\title{
Genome Analysis of the Fatal Tapeworm Sparganum Proliferum Uncovers the Cryptic Life Cycle and Mechanisms Underlying Aberrant Larval Proliferation
}

Taisei Kikuchi ( $\nabla$ taisei_kikuchi@med.miyazaki-u.ac.jp )

University of Miyazaki https://orcid.org/0000-0003-2759-9167

Mehmet Dayi

University of Miyazaki

Vicky Hunt

University of Bath

Atsushi Toyoda

National Institute of Genetics https://orcid.org/0000-0002-0728-7548

Yasunobu Maeda

University of Miyazaki

Yoko Kondo

Faculty of Medicine, Tottori University

Belkisyole Alarcon de Noya

Central University of Venezuela

Oscar Noya

Central University of Venezuela

\section{Somei Kojima}

Chiba-Nishi General Hospital, Matsudo City, Chiba

\section{Toshiaki Kuramochi}

National Museum of Nature and Science, 4-1-1 Amakubo, Tsukuba

Haruhihko Maruyama

University of Miyazaki

\section{Article}

Keywords: pseudophyllidean tapeworm, gene family evolution, relaxed selection, extracellular matrix coordination, asexual reproduction, oncogenes, homeobox, fibronectin, cadherin

Posted Date: September 25th, 2020 
DOI: https://doi.org/10.21203/rs.3.rs-78313/v1

License: (c) (1) This work is licensed under a Creative Commons Attribution 4.0 International License. Read Full License

Version of Record: A version of this preprint was published at Communications Biology on May 31st, 2021. See the published version at https://doi.org/10.1038/s42003-021-02160-8. 
1 Genome analysis of the fatal tapeworm Sparganum proliferum uncovers the cryptic life cycle and

2 mechanisms underlying aberrant larval proliferation

3

4 Taisei Kikuchi ${ }^{*}$, Mehmet Dayi ${ }^{1,2}$, Vicky L. Hunt ${ }^{3}$, Atsushi Toyoda ${ }^{4}$, Yasunobu Maeda ${ }^{1}$, Yoko

$5 \mathrm{Kondo}^{5}$, Belkisyole Alarcon de Noya ${ }^{6}$, Oscar Noya ${ }^{6}$, Somei Kojima ${ }^{7}$, Toshiaki Kuramochi ${ }^{8}$,

6 Haruhiko Maruyama ${ }^{1}$

7

$8{ }^{1}$ Faculty of Medicine, University of Miyazaki, Miyazaki, 889-1692 Japan

9 2Forestry Vocational School, Duzce University, 81620, Duzce, Turkey

103 Department of Biology and Biochemistry, University of Bath, Bath, BA27AY UK

$11{ }^{4}$ Comparative Genomics Laboratory, Department of Genomics and Evolutionary Biology, National

12 Institute of Genetics, Mishima, Shizuoka, 411-8540 Japan

13 5Division of Medical Zoology, Department of Microbiology and Immunology, Faculty of Medicine,

14 Tottori University, Yonago 683-8503, Japan

$15{ }^{6}$ Institute of Tropical Medicine, Central University of Venezuela, Venezuela, 2101 Maracay, 16 Caracas, Venezuela

$17{ }^{7}$ Department of Clinical Laboratory Medicine, Chiba-Nishi General Hospital, Matsudo City, Chiba, 18 270-2251 Japan

19 8Department of Zoology, National Museum of Nature and Science, 4-1-1 Amakubo, Tsukuba, 20 Ibaraki, 305-0005 Japan

21

22

23 *Corresponding author;

24 Taisei Kikuchi, Division of Parasitology, Faculty of Medicine, University of Miyazaki, Miyazaki 88925 1692, Japan, Tel: +81-985850990, Fax: +81-985843887, email: taisei kikuchi@med.miyazaki26 u.ac.jp 
The cryptic parasite Sparganum proliferum proliferates in humans and invades tissues and organs.

31 Only scattered cases have been reported, but $S$. proliferum infection is always fatal. However, $S$. proliferum's phylogeny and life cycle remain enigmatic. To investigate the phylogenetic relationships between $S$. proliferum and other cestode species, and to examine the mechanisms underlying pathogenicity, we sequenced the entire genomes of $S$. proliferum and a closely related non-life-threatening tapeworm Spirometra erinaceieuropaei. Additionally, we performed larvae transcriptome analyses of $S$. proliferum plerocercoid to identify genes involved in asexual reproduction in the host. The genome sequences confirmed that the $S$. proliferum has experienced a clearly distinct evolutionary history from $S$. erinaceieuropaei. Moreover, we found that nonordinal extracellular matrix coordination allows asexual reproduction in the host, and loss of sexual maturity in $S$. proliferum are responsible for its fatal pathogenicity to humans. Our high-quality reference genome sequences should be valuable for future studies of pseudophyllidean tapeworm biology and parasitism.

Keywords: pseudophyllidean tapeworm, gene family evolution, relaxed selection, extracellular matrix coordination, asexual reproduction, oncogenes, homeobox, fibronectin, cadherin

\section{Background}

The cryptic parasite Sparganum proliferum was first identified in a 33-year-old woman in Tokyo in 1904. The patient's skin was infected with numerous residing cestode larva of unknown taxonomy. ljima et al [1] originally designated the parasite as Plerocercoides prolifer, and considered it a pseudophyllidean tapeworm in the plerocercoid larval stage. In 1907, an extremely similar human case was reported by Stiles in Florida, USA, and the responsible parasite was renamed $S$. proliferum [2]. Clinical symptoms and post-mortem findings indicate that $S$. proliferum proliferates in humans and invades various organs and tissues, including the skin, body walls, lungs, abdominal viscera, lymph nodes, blood vessels, and the central nervous system, leading to miserable disease prognosis $[3,4]$. Not many cases have been reported to date but the infection was fatal in all reported cases (reviewed in [5]).

58 There was a postulation about the origin of this parasite. Some parasitologists considered it to be a new species of pseudophyllidean tapeworm, whereas others suspected that $S$. proliferum was a virus-infected or aberrant form of Spirometra erinaceieuropaei, based on morphological similarities $[6,7]$. Recent DNA sequence analyses of mitochondrial NADH dehydrogenase subunit III, mitochondrial tRNA, cytochrome oxidase subunit I, and nuclear succinate dehydrogenase ironsulfur protein subunit ( $\mathrm{sdhB}$ ) genes suggested that $S$. proliferum is a closely related but distinct 
observed and the precise taxonomic relationships of $S$. proliferum with other worms remain unclear because few genes have been sequenced.

In addition to taxonomic considerations, the pathogenicity of $S$. proliferum and its mechanisms of proliferation and invasion in mammalian hosts are of considerable interest. In principle, plerocercoids of pseudophyllidean tapeworms (spargana), including those of $S$. erinaceieuropaei and other Spirometra species, do not proliferate asexually, but migrate through subcutaneous connective tissues, causing only non-life threatening sparganosis (non-proliferative sparganosis). Other organs, such as the lungs and liver or the central nervous system, may be niches for these worms but are not commonly described. Symptoms of non-proliferative sparganosis are mainly caused by the simple mass effect [5].

Asexual proliferation of larvae and the destruction of host tissues are characteristic of cyclophyllidean tapeworms such as Echinococcus, which proliferates asexually by generating a peculiar germinative layer in a hydatid cyst form [10]. In another cyclophyllidean tapeworm, Mesocestoides, asexual multiplication is achieved by longitudinal fission [10,11]. In contrast, the pseudophyllidean Sparganum plerocercoid undergoes continuous branching and budding after invading the human body by an unidentified route, and produces vast numbers of progeny plerocercoids.

To clarify the phylogenetic relationship of the enigmatic parasite $S$. proliferum with other cestode species and investigate the underlying pathogenic mechanisms, we sequenced its entire genome as well as that of newly isolated $S$. erinaceieuropaei. We also performed transcriptome analyses of $S$. proliferum plerocercoid larvae to identify genes that are involved in asexual reproduction in the host. Those analyses revealed its phylogeny and gene evolution that contribute to the proliferation and pathogenicity of $S$. proliferum.

\section{Results}

Genomic features of $S$. proliferum and S. erinaceieuropaei

We sequenced the $S$. proliferum genome using multiple insert-length sequence libraries (Additional Table S1) and compiled a $653.4-\mathrm{Mb}$ assembly of 7388 scaffolds with N50 of $1.2 \mathrm{Mb}$. The S. erinaceieuropaei genome was assembled into $796 \mathrm{Mb}$ comprising 5723 scaffolds with N50 of $821 \mathrm{~kb}$. These assembly sizes were $51.9 \%$ and $63.2 \%$ of the previously published $S$. erinaceieuropaei genome (UK isolate) [12]. CEGMA and BUSCO report the percentage of highly conserved eukaryotic gene families that are present as full or partial genes in assemblies and nearly $100 \%$ of core gene families are expected in most eukaryote genomes. BUSCO analyses showed that $88.1 \%$ and $88.5 \%$ of core gene families were represent in $S$. proliferum and $S$. erinaceieuropaei genomes, respectively, higher than or comparable to other previously published tapeworm genomes (Table 1). CEGMA completeness values for $S$. proliferum and $S$. erinaceieuropaei were slightly lower than those from BUSCO analyses. Low CEGMA completeness was also seen in other pseudophyllidea tapeworm genomes, including $S$. 

CEGMA completeness values of these two genome assemblies, therefore, indicate pseudophyllidean-specific loss or high divergence of the genes that are conserved in other eukaryotic taxa. The average numbers of CEGs (hits for 248 single-copy eukaryotic core genes) for $S$. proliferum and $S$. erinaceieuropaei were 1.2 and 1.3, respectively, indicating that the assembly sizes roughly represent the haploid genome sizes of these tapeworms. However, in Kmer analyses of Illumina short reads, we estimated haploid genome sizes of 582.9 and $530.1 \mathrm{Mb}$ for S. proliferum and S. erinaceieuropaei, respectively (Additional Fig S1a), indicating that the assemblies contain heterozygous haplotypes and/or overestimated gap sizes. Ploidies were inferred from heterozygous K-mer pairs and were diploid for both species (Figure S1b).

113 The genomes of $S$. proliferum and S. erinaceieuropaei are highly repetitive, with about $55.0 \%$ 114 repetition of the total genome length in both genomes (Additional Fig S2 and Table 2). Long 115 interspersed nuclear elements (LINEs) occupy $26.3 \%$ and $31.9 \%$ of the total genomes of $S$. 116 proliferum and $S$. erinaceieuropaei, respectively. These LINEs predominantly comprise the three 117 types (Penelope, RTE-BovB, and CR1), which are also abundant in other pseudophyllidea 118 genomes (Additional Fig S2).

119 A total of 25627 genes were predicted in $S$. proliferum assemblies, about 5000 fewer than for $S$. 120 erinaceieuropaei (30751), but more numerous than for other cestode genomes. In studies of the 121 S. erinaceieuropaei UK isolate \{Bennett, 2014 \#39\}, the gene number (> 39000) was likely overestimated due to fragmentation and redundancy in the assembly.

124 Phylogenetic placement of S. proliferum

125 Phylogenetic relationships of S. proliferum with other cestode species were inferred from 205 126 single-copy orthologues (Figure 1). A clear separation was identified between pseudophyllidea and 127 cyclophilidea clades. In the pseudophyllidea clade, S. proliferum occupied the basal position of the 128 Spirometra cluster, in which two S. erinaceieuropaei isolates (Japan and UK isolates) were placed 129 beside each other.

130 Phylogenetic tree topology based on mitogenomes of the 14 cestodes and all available 131 mitogenome data of Spirometra in the GenBank, was similar to that of the nuclear genome 132 (Additional Fig S3). Yet in contrast with the nuclear genome tree, the S. erinaceieuropaei UK 133 isolate was located in a basal position of the Spirometra cluster, placing S. proliferum in the middle 134 of Spirometra species, albeit with a long branch. These inconsistencies between nuclear and 135 mitogenome trees may reflect uncertainties of species classification in the genus Spirometra [13, 136 14]. Moreover, mitochondrial sequences can give poor inferences of species trees [15]. 137 Cumulatively, these results suggest that $S$. proliferum has a close phylogenetic relationship with 138 Spirometra but is clearly distinguished by genomic features and gene contents. 
141 Protein family (Pfam) analyses revealed highly similar protein domain distributions of $S$. proliferum

142 and Spirometra genomes ( $r=0.99$; Figure 2, Additional Table S2). Few domains differed

143 significantly in abundance between the two species. Among these, the $S$. proliferum genome was 144 underrepresented in zinc-finger families ( $\mathrm{Zf}-\mathrm{C} 2 \mathrm{H} 2$, Zf-C2H2_4, Zf-C2H2_6, Zf-C2H2_jaz and Zf145 met), reverse transcriptase (RVT_1), exo/endonuclease/phosphatase, galactosyltransferase, and 146 alpha/beta hydrolase (abhydrolase_6). Overrepresented Pfam domains in S. proliferum included 147 a distinct type of zinc-finger domain ( $z f-3 C x x C$ ), fibronectin type III (fn3), trypsin, RNA polymerase 148 III RPC4 (RNA_pol_Rpc4), and an ADP-specific phosphofructokinase/glucokinase conserved 149 region (ADP_PFK_GK).

150 We performed gene family analysis using OrthoFinder with the predicted proteomes of $S$. 151 proliferum, S. erinaceieuropaei, and other selected cestode genomes. A total of 234522 proteins 152 from 14 cestode species were placed into 39174 gene families (Figure 1). The $S$. proliferum 153 proteome (25627 proteins) was encoded by 9136 gene families, among which 7364 were shared 154 by all 14 cestodes and 2550 proteins were specific to the species or singleton. The $S$. 155 erinaceieuropaei proteome (30751 proteins) was clustered into 9008 gene families, 3806 of which 156 were species specific or singletons. Only four gene families were specific to both Spirometra and 157 Sparganum.

158 We used computational analysis of gene family evolution (CAFE) to estimate gene family 159 expansion and contraction, and identified gene families with significantly higher than expected 160 rates of gains and losses (Figure 3, Additional Table S3). Twenty-one gene families were 161 significantly expanded in the $S$. proliferum lineage, and these included annotations for fibronectin, 162 reverse transcriptase, zinc-finger $\mathrm{C} 2 \mathrm{H} 2$ type, and core histone (Additional Table S4). Significantly 163 contracted gene families (43 families) had annotations relating to signal transduction proteins, such 164 as phosphatases and kinases, and ion channels and ABC transporters (Additional Table S5). 165 Fibronectin, reverse transcriptase, zinc-finger $\mathrm{C} 2 \mathrm{H} 2$ type, and peptidases were present in 166 expanded and contracted families.

167 In the $S$. erinaceieuropaei lineage, 63 and 15 gene families were significantly expanded or 168 contracted (Additional Table S6 and S7), respectively. Among them, highly lineage specific 169 expansion was found for 7 families (i.e. 10 or more genes in S. erinaceieuropaei, whereas one or 170 no genes in S. proliferum. For example, the Orthogroup OG0000184 contains one S. proliferum 171 gene and 44 S. erinaceieuropaei genes, encoding biphenyl hydrolase-like protein (BPHL), which 172 harbors the Pfam domain abhydrolase_6 (Figure 2). Although the other gene families mostly 173 encode proteins of unknown function, they were likely expanded after speciation from S. proliferum 174 and $S$. erinaceieuropaei and may have specific roles in the $S$. erinaceieuropaei lifecycle or 175 parasitism.

176

177 Conserved developmental pathway genes 
178 Homeobox transcription factors are involved in patterning of body plans in animals. The homeobox

179 gene numbers are much fewer in parasitic flatworms than in most other bilaterian invertebrates,

180 which have a conserved set of approximately 100 homeobox genes. Genome severance of four

181 cyclophyllid cestodes revealed that out of 96 homeobox gene families that are thought to have

182 existed at the origin of the bilateria, 24 are not present in cestodes [16]. The pseudophyllid

183 cestodes S. proliferum and S. erinaceieuropaei have similar homeobox class repertoires as those

184 in cyclophyllid cestodes, in which class ANTP was the most abundant, followed by classes PRD

185 and TALE; Table 3). The total numbers of homeobox domains identified in S. proliferum and $S$.

186 erinaceieuropaei are 64 and 71, respectively, and because these were fewer than in the

187 cyclophyllids Echinococcus multilocularis and Taenia solium (Table 3), they are the most reduced

188 of any studied bilaterian animal. The three homeobox families Pou/Pou6, ANTP/Bsx, and

189 ANTP/Meox were not present in S. proliferum and S. erinaceieuropaei, whereas the homeobox

190 family ANTP/Ro was found in S. proliferum and S. erinaceieuropaei but not in E. multilocularis and

191 T. solium (Additional Fig S4).

192 Comparisons between S. proliferum and S. erinaceieuropaei showed that the homeobox families

193 TALE/Pknox, ANTP/Hox1, ANTP/Msxlx, and POU/Pou-like are missing in S. proliferum, despite

194 being present in the other cestodes. In contrast, the homeobox families ANTP/Dbx and PRD/Alx

195 were found in S. proliferum but not in S. erinaceieuropaei.

196 Other conserved genes with roles in flatworm developmental pathways, such as Hedgehog and

197 Notch, were conserved in S. proliferum and S. erinaceieuropaei. But in the Wnt pathway, whose

198 complement is much smaller than the ancestral complement in tapeworms [16], two further genes

199 (Axin and LEF1/TCF) were missing in S. proliferum and S. erinaceieuropaei (Table S8).

200

201 Horizontally transferred genes

202 To determine whether the present genomes contained horizontally transferred genes (HTGs) from

203 other organisms, we used a genome-wide prediction method based on a lineage probability index

204 using the software Darkhorse2 identified 19 and 33 putative HTGs in S. proliferum and $S$.

205 erinaceieuropaei, respectively (Additional Table S9 and S10). For these transfers, all possible host

206 organisms were bacteria except for one Spirometra gene that has high similarity to a chlorella virus

207 gene. Orthologues of most $S$. proliferum putative HTGs were also detected as horizontally

208 transferred in S. erinaceieuropaei. Moreover, possible host bacteria, including Marinifilum breve,

209 Aphanizomenon flos-aquae, Alcanivorax sp., and Vibrio sp., were shared by the two cestode

210 species and were aquatic or marine bacteria, indicating that these genes were acquired by a

211 common ancestor of the two tapeworms which had aquatic phase in the life cycle.

212

213 Positive selection of the S. proliferum lineage

214 Positive selection is a mechanism by which new advantageous genetic variants sweep through a

215 population and drive adaptive evolution. To investigate the roles of positive selection in the 
216 evolution of $S$. proliferum, we performed $\mathrm{dN} / \mathrm{dS}$ branch-site model analyses with single-copy

217 orthologous genes from 12 tapeworms and identified a total of 35 positively selected genes in the

218 S. proliferum lineage (Additional Table S11). Evolutionary pressures were identified for some

219 genes that are essential to cellular processes, including transcription/RNA processing/translation

220 genes encoding DNA-directed RNA polymerase II subunit, polypyrimidine tract-binding protein,

221 adenylate kinase, ribosomal protein L21, snu13 NHP2-like protein, and eukaryotic translation

222 initiation factors. Other identified genes were related to transportation (dynein intermediate chain

223 2) and mitochondrial processes (Rieske). Genes involved in stress and immune responses, such

224 as DNAJ/Hsp40, HIKESHI protein, Toll-like receptor, and Ig_3/lg, were also positively selected in

225 the S. proliferum lineage, along with the RAS oncogene Rab-4A.

226 Environmental change often eliminates or weakens selective pressures that were formerly 227 important for the maintenance of a particular trait [17]. We detected 9 genes that were subject to 228 these circumstances of "relaxed selection" in the $S$. proliferum lineage, relative to the other 229 tapeworm lineages (Additional Table 12). These genes encode proteins with putative roles in 230 developmental regulation and cell differentiation. In particular, the receptor roundabout (ROBO) 231 and secreted molecules of the SLIT family, together, play important roles in guiding axons and 232 proper morphogenesis [18]. The Rho GTPase-activating protein is also highly expressed in highly 233 differentiated tissues and affects cell differentiation by negatively regulating Rho-GTPase signaling 234 [19]. Delta-like protein (DLL) is an inhibitory ligand of the Notch receptor pathway and is expressed 235 during brain development [20]. Vascular endothelial growth factor receptor is also known to 236 regulate stem cell homeostasis and repopulation in planarian species [21]. Hence, these instances 237 of relaxed selection indicate that the worm has long since used certain developmental pathways. 238 We also identified two genes encoding cadherin (protocadherin) that were subject to relaxed 239 selection. Cadherein is a transmembrane protein that mediates cell-cell adhesion in animals and 240 those relaxed selections indicate diverging cell adhesion process in the worm.

242 Differential gene expression involved in asexual proliferation and parasitism

243 We maintained $S$. proliferum via serial infection of mice and found that some plerocercoid worms 244 exhibit a highly branching structure (medusa-head form; Figure 4a), which was observed 245 frequently in heavily infected mice. In contrast, in mice with low worm burdens, most worms had 246 unadorned non-branching morphology (wasabi-root form). Worms with the medusa-head form are 247 considered the main sources of new plerocercoid worms in the host, and their proliferation is highly 248 related to their pathogenicity. We, therefore, identified genes with expression levels that 249 distinguished medusa-head and wasabi-root forms.

250 RNAseq analysis revealed 357 differentially expressed genes (DE genes) between medusa-head 251 and wasabi-root forms (246 upregulated and 111 downregulated in medusa-head) (Figure 4b). 252 The upregulated set in medusa-head forms were dominated by genes encoding peptidases and 253 peptidase inhibitors, such as tolloid-like proteins (19 genes), chymotrypsin-like proteins (6 genes) 
and CAP domain-containing proteins (12 genes) as well as transposon-related proteins such as gag-pol polyproteins and reverse transcriptases (30 genes) (Additional Table S13). This set of DE genes was enriched in the GO categories for metalloendopeptidase activity and proteolysis (Additional Table S14). Downregulated genes also encoded a variety of peptidases and peptidase inhibitors, including leucyl aminopeptidase (5 genes), chymotrypsin-like elastase (7 genes), and kunitz bovine pancreatic trypsin inhibitor domain protein (3 genes), with high representation under the GO terms metalloexopeptidase, aminopeptidase, and manganese ion binding (Additional Table S14). Peptidases and peptidase inhibitors are secreted by many types of pathogens, including bacteria, fungi, and parasites, and often play critical roles in survival and virulence [2224].Other genes known to be involved in pathogenicity in other pathogens were also upregulated in the medusa-head form, including genes encoding multidrug resistance-associated proteins [25] and tetraspanins. The latter proteins have four transmembrane domains and not only play roles in a various aspects of cell biology but also are used by several pathogens for infection and regulate cancer progression [26].

Genes that are involved in cell-growth and cancer development were also upregulated in the medusa-head form, including those encoding proteins from wnt (wnt-111 and wnt-5) and ras/rab (ras-0b, ras-2 and Rasef) pathways, transcription factors/receptors (sox1a, fibroblast growth factor receptor) and homeobox proteins (prospero, PAX, orthopedia ALX and ISL2).

It has been shown that expansions of gene families and changes in expression levels have been associated with the evolution of parasitism in previous studies [27, 28]. An upregulation of genes from expanded gene families was also found in $S$. proliferum. For instance, 15 genes were identified as upregulated from an expanded gene family (OrthoGroup OG0000040). The orthogroup OG0000044 includes genes encoding mastin precursors, and six of these were upregulated and another six were downregulated in the medusa-head form (Additional Table S13). Phylogenetic analyses of those gene families indicate that some of these orthogroups are conserved across flatworms, while others are specific to the Pseudophillidea clade of flatworms (Additional Fig S5).

Among the present DE genes, 85 that were upregulated in medusa-head forms have no known functions. These included 17, 10, 3, 2, and 2 genes from orthogroups OG0000083, OG0003096, OG0010117, OG0011363, and OG0011373, respectively. These orthogroups were expanded in the S. proliferum lineage and the DE genes had extremely high fold changes (Figure 4c). Because their products predominantly harbour secretion signal peptides (Additional Table S13), they are likely to be secreted by the parasite into the host and play important roles in parasitism, aberrant larval proliferation in the host, and/or modulation of host immunity.

\section{Discussion}


291 S. proliferum is a cryptic parasite with fatal consequences, but its phylogeny and lifecycle are

292 poorly understood. In this study, we sequenced the $S$. proliferum genome and performed 293 comparative genomics with other tapeworm species, including the newly-sequenced $S$. 294 erinaceieuropaei genome. The S. erinaceieuropaei genome was sequenced previously [12], with 295 an estimated genome size of more than $1.2 \mathrm{~Gb}$, but because the source material was from a biopsy 296 the assembled sequence was highly fragmented. Hence, the S. erinaceieuropaei genome 297 presented herein provides a more reliable estimate of the size and contents of this parasite 298 genome. The new genome assembly was about two thirds of the size of the previous assembly 299 but remains the largest genome among sequenced tapeworms. Compared to cyclophyllidean 300 tapeworms, including Echinococcus and Taenia spp., for which high-quality genome references 301 are available [16, 29, 30], genome information for pseudophyllidean tapeworms is limited [31]. The 302 genomes presented in this study could, therefore, serve as a powerful resource for more 303 comprehensive studies of tapeworm genomics and will facilitate the understanding of 304 pseudophyllidean tapeworm biology and parasitism.

305

306 There have been three big knowledge gaps for the present cryptic tapeworm: 1) its phylogenetic 307 relationship with Spirometra species, 2) its lifecycle including the definitive and intermediate hosts, 308 and 3) genetic and physiological differences with non-proliferating Spirometra species that enable 309 the worm to reproduce asexually in non-definitive hosts, such as humans and mice.

310 To determine phylogenetic relationships, we confirmed that the genetic sequence of $S$. proliferum 311 is distinct from that of $S$. erinaceieuropaei, despite the close relationship between these species. 312 Specifically, the $S$. proliferum genome is about 150-Mb smaller and contains 5000 fewer protein 313 coding genes than in S. erinaceieuropaei. Both genomes, nonetheless, showed diploidy. These 314 data suggest that $S$. proliferum is not an aberrant form of Spirometra worm by virus infection or by 315 small mutations [6, 7] and not a hybrid origin of multiple Spirometra species. In agreement, no 316 virus-like sequences were detected in S. proliferum DNA or RNA raw reads.

317 We were unable to identify definitive or intermediate hosts of $S$. proliferum in the current study. 318 Recent horizontal transfers of genes or mobile elements can indicate phylogenetic relationships, 319 because HGT events occur between closely associated organisms. Well-known examples include 320 HGT from Wolbachia symbionts to their host insect [32, 33] and transfer of BovB retrotransposons 321 between ruminants and snakes via parasitic ticks [34, 35]. We found that RTE/BovB repeats are 322 abundant in the $S$. proliferum genome, but were likely acquired by an ancestral pseudophyllidea, 323 as indicated by their abundance in D. latum and S. solidus. Moreover, our HGT screening analyses 324 indicate several genes that were likely acquired from bacteria but these HGTs likely have occurred 325 before specification of $S$. proliferum and $S$. erinaceieuropaei. The high-quality reference genomes 326 presented herein, however, provide valuable resources for further attempts to identify vestigial $S$. 327 proliferum sequences in other organisms or to perform analyses of protein-protein interactions 328 between hosts and parasites. 
329 Loss of genes that are involved in the development of multicellular organisms and nervous systems,

330 including homeobox genes and genes for zinc-finger domain containing proteins, and relaxed

331 selection of some developmental genes (ROBO, Slit, RHOGAP, etc.) suggests that $S$. proliferum

332 has lost the ability to undergo proper development and complete the sexual lifecycle. Although the

333 precise functions of homeobox genes in tapeworms remain elusive, proteins of homeobox families

334 that are missing in S. proliferum (TALE/Pknox, ANTP/Hox1, ANTP/Msxlx and POU/Pou-like)

335 appear to have important roles in the development of embryos and adult body plans. For example,

336 Hox1 of the HOX gene family specifies regions of the body plan of embryos and the head-tail axis

337 of animals [36]. Products of the Pknox gene family, also known as the PREP gene family, are

338 implicated as cofactors of Hox proteins [37]. Msxlx homeobox gene was highly upregulated in the

339 ovaries and was continually expressed in fertilized ova in the uterus in Hymenolepis microstoma.

340 This gene was related to the female reproductive system in this tapeworm [38]. POU class genes

341 are present in all animals and are extensively in nervous system development and the regulation

342 of stem cell pluripotency in vertebrates [39]. Specific loss of Pou-like genes and relaxed selection

343 of Pou3 suggest that $S$. proliferum has low dependency on POU genes.

344 We contend that the loss of sexual maturity of this parasite is related to its fatal pathogenicity in

345 humans, because survival of the parasite is dependent on asexual reproductive traits of budding

346 and branching, which lead to $100 \%$ lethality in infected humans. Accordingly, we identified genes

347 that are upregulated in vigorously budding worms using transcriptome analyses and then selected

348 genes that are putatively important for asexual proliferation, such as a variety of peptidase genes

349 and oncogene-like genes. Among them, groups of secreted proteins with unknown functions were

350 of great interest. They were expanded in the S. proliferum genome and showed more than 10-fold

351 changes in expression levels. Recently, an S. erinaceieuropaei gene belonging to one of those

352 groups (orthogroup OG0000083) was cloned and named plerocercoid-immunosuppressive factor

353 (P-ISF) (Yoko Kondo, under review). P-ISF is a cysteine-rich glycoprotein abundant in plerocercoid

354 excretory/secretory products and likely involved in immunomodulation of its hosts by suppressing

355 osteoclastgenesis including the gene expression of TNF- $\alpha$ and IL-1 $\beta$, and nitric oxide production

356 in macrophages [40, 41]. Upregulation of P-ISF genes in S. proliferum proliferating worms is

357 therefore reasonable and the expansion of the gene family in $S$. proliferum indicates the

358 considerable contribution to the specific lifecycle. The other upregulated gene families of unknown

359 function are also expanded in S. proliferum suggesting possible important roles in the hosts,

360 therefore, future studies of these novel genes are required to fully understand the mechanism

361 underlying the $S$. proliferum parasitism.

362 Fibronectin is an extracellular matrix (ECM) glycoprotein that controls the deposition of other ECM

363 proteins, including collagens and latent TGF-beta binding protein [42]. During branching

364 morphogenesis, accumulations of fibronectin fibrils promote cleft formation by suppressing

365 cadherin localization, leading to loss of cell-cell adhesion [43]. The present observations of the $S$.

366 proliferum lineage show specific expansions of three gene families containing fibronectin type III 
domains. S. proliferum also had fewer cadherin genes than S. erinaceieuropaei and three of them are subject to relaxed selection in S. proliferum. These results collectively suggest nonordinal ECM coordination in S. proliferum, allowing the formation of highly branching structures and enabling asexual proliferation in the host.

371

372

\section{Methods}

374 Biological materials

375 S. proliferum strain Venezuela was used for the genome analyses. The parasite was originally 376 isolated from a Venezuelan patient in 1981 and has been maintained by serial passages using

377 BALB/c mice via intraperitoneal injections of the plerocercoids in National Science Museum as 378 described in Noya et al $[44,45]$. S. erinaceieuropaei was isolated from a Japanese four-striped rat 379 snake (Elaphe quadrivirgata) collected in Yamaguchi prefecture, Japan in 2014.

380

381 DNA and RNA extraction and sequencing

382 S. proliferum worms were collected from the abdominal cavity of infected mice and washed 383 thoroughly with $1 \times$ PBS. Plerocercoids of $S$. erinaceieuropaei were isolated from the subcutaneous 384 tissues of the snake. Genomic DNA was extracted using Genomic-tip (Qiagen) following the 385 manufacturer's instructions.

386 Paired-end sequencing libraries (Additional Table 1) were prepared using the TruSeq DNA Sample 387 Prep kit (Illumina) according to the manufacturer's instructions. Multiple mate-paired libraries (3, 8, 38812 and $16 \mathrm{~kb}$ ) were also constructed using the Nextera Mate-Paired Library Construction kit 389 (Illumina). Libraries were sequenced on the Illumina HiSeq 2500 sequencer using the Illumina 390 TruSeq PE Cluster kit v3 and TruSeq SBS kit v3 (101, 150 or 250 cycles $\times 2)$ or the Illumina MiSeq 391 sequencer with the v3 kit (301 cycles $\times 2$ ) (Additional Table S1). The raw sequence data were 392 analysed using the RTA 1.12.4.2 analysis pipeline and were used for genome assembly after 393 removal of adapter, low quality, and duplicate reads.

394 RNA was extracted from individual worms using TRI reagent according to the manufacturer's 395 instructions. Total RNA samples were qualified using Bioanalyzer 2100 (Agilent Technology, Inc.). 396 Only samples with an RNA integrity value (RIN) greater than 8.0 were used for library construction. 397 One hundred ng of total RNA was used to construct an Illumina sequencing library using the 398 TruSeq RNA-seq Sample Prep kit according to the manufacturer's recommended protocols 399 (Illumina, San Diego, USA). The libraries were sequenced for 101 or 151 bp paired-ends on an 400 Illumina HiSeq2500 sequencer using the standard protocol (Illumina).

401

402 K-mer Analysis 
403 A k-mer count analysis was performed using K-mer Counter (KMC) [46], on the paired-end Illumina

404 data. Only the first read was used to avoid counting overlapping k-mers. Genome size and ploidy

405 estimations were performed using Genomescope [47] and Smudgeplot, respectively [48].

406

407 Genome assembly

408 Illumina reads from multiple paired-end and mate-pair libraries (Additional Table 1) were 409 assembled using the Platanus assembler [49] with the default parameter. Haplomerger2 [50] was 410 then used to remove remaining haplotypic sequences in the assembly and contigs were further 411 scaffolded using Illumina mate-pair reads using SSPACE [51]. CEGMA v2 [52] and BUSCO [53] 412 were used to assess the completeness of the assemblies.

413 Mitochondrial genomes (mitogenomes) were reconstructed from Illumina reads with MITObim 414 version 1.6 [54]. Mitochondrial fragments in the nuclear genome assembly were identified by 415 BLASTX using S. mansonai mitochondrial genes as queries and those fragments were extended 416 by iterative mappings of Illumina short reads using MITObim. Assembled mitogenomes were 417 annotated for protein-coding, tRNA and rRNA genes using the MITOS web server [55]. Assemblies 418 and annotations were manually curated using the Artemis genome annotation tool [56] with based 419 on evidence supports from sequence similarity to other published mitogenomes.

420

421 Repeat analysis

422 Repeats within the genome assemblies were identified using RepeatModeler (v1.0.4, 423 http://www.repeatmasker.org/RepeatModeler.html) and RepeatMasker (v.3.2.8, $424 \mathrm{http} / / /$ www.repeatmasker.org) to calculate the distribution of each repeat and its abundance in the 425 genome.

426

427 Gene prediction and functional annotation

428 To predict protein-coding genes, Augustus (v. 3.0.1) [57] was trained for S. proliferum and $S$. 429 erinaceieuropaei, individually, based on a training set of 500 non-overlapping, manually curated 430 genes. To obtain high-confidence curated genes, a selection of gene models from gene predictions 431 based on Augustus S. mansonai parameters, were manually curated in Artemis using aligned 432 RNA-seq data and BLAST matches against the NCBI database. RNA-seq reads were mapped to 433 the genomes using Hisat2 (parameters: --rna-strandness RF --min-intronlen 20 -max-intronlen 434 10000) [58]. Based on the Hisat2 alignments, the bam2hints program (part of the Augustus 435 package) was used to create the intron hints, with minimum length set to $20 \mathrm{bp}$. Augustus were 436 run with trained parameters using all the hints for that species as input. Introns starting with 'AT' 437 and ending with 'AC' were allowed (--allow_hinted_splicesites=atac). A weight of $10^{5}$ was given to 438 intron and exonpart hints from RNA-seq. If Augustus predicted multiple, alternatively spliced 439 transcripts for a gene, we only kept the transcript corresponding to the longest predicted protein 440 for further analyses. 
441 Functional annotations were performed on the gene models based on multiple pieces of evidence

442 including BLASTP search against NCBI nr database and the latest version Pfam search (ver. 30.0)

443 with HMMER3 [59]. Gene ontology (GO) terms were assigned to genes using Blast2Go (v2) [60]

444 with BLAST search against NCBI nr database and the InterProScan results.

445

446 Species tree reconstruction

447 Amino acid sequences in each single-copy gene family were aligned using MAFFT version 448 v7.22152 [61], poorly aligned regions were trimmed using GBlocks v0.91b53 [62], and then the 449 trimmed alignments were concatenated. A maximum-likelihood phylogenetic tree was produced 450 based on the concatenated alignment using RAxML v8.2.754 [63] with 500 bootstrap replicates. 451 The best-fitting substitution model for each protein alignment was identified using the RAxML 452 option (-m PROTGAMMAAUTO). Mitochondrial genome phylogeny was also constructed by the 453 same method using 12 protein coding genes on mitogenomes.

454

455 Gene family analysis

456 To estimate branch or lineage specific gain and loss of orthologous gene families, OrthoFinder 457 [64] and CAFÉ (v3) [65] under parameters “-p 0.01, -r 1000" were used.

458

459 Screening for horizontally transferred genes

460 To screen potential horizontal gene transfers (HGTs) into the S. proliferum and S. erinaceieuropaei 461 lineages, we used DarkHorse v2, which detects phylogenetically atypical proteins based on 462 phylogenetic relatedness of blastp hits against a taxonomically diverse reference database using 463 a taxonomically-weighted distance algorithm [66]. Options (-n 1 -b 0.5 -f 0.1 ) were used in 464 DarkHorse HGT screening.

465

466 Positive Selection Scans (dNdS)

467 To analyse selection pressures in S. proliferum genes, the ETE3 Python package [67] for CODEML 468 [68] was employed to calculate the non-synonymous (dN) and synonymous (dS) substitutions 469 rates, and the ratio ( $\mathrm{dN} / \mathrm{dS}$ or $\omega$ ). Nucleotide sequences of single copy orthologue genes from 12 470 cestode species (S. proliferum, S. erinaceieuropaei, Diphyllobothrium latum, Schistocephalus 471 solidus, Hymenolepis diminuta, Hymenolepis nana, Hydatigera taeniaeformis, Taenia solium, 472 Taenia asiatica, Echinococcus multilocularis, Echinococcus granulosus, Mesocestoides corti) 473 were aligned based on amino acid alignment using Pal2aln v14 [69] with the parameters (474 nomismatch and-nogap). dN/dS were estimated using branch-site models with $S$. proliferum as 475 the foreground and other branches in the tree as the background. The non-null model (bsA) were 476 compared with the null model (bsA1) for each tree using a likelihood ratio test (LRT), where log477 likelihood ratios were compared to a chi-square distribution with 1 degree of freedom. False 
discovery rate (FDR) correction were performed over all the P-values and genes showing FDR $<0.05$ were manually curated before obtaining final $\mathrm{dN} / \mathrm{dS}$ values.

480 Test for relaxed selection was performed using the RELAX tool [70] with aforementioned single copy orthologue gene sets. The relaxation parameter $\mathrm{k}$ was calculated for each blanch and tested by LRT with $S$. proliferum as foreground and the others as background.

483

RNAseq analysis

For gene expression analyses, S. proliferum plerocercoid worms were grouped into two types based on the morphology and proliferation activity; worms vigorously branching to form structure like "Medusa head" and worms under static form to form like "Wasabi root" (Figure 4a). Worms were collected from infected mice on $\sim 50$ weeks post inoculation. RNA was extracted from the individual worms and sequenced as described above. RNAseq reads were mapped to the $S$. proliferum reference genomes (v2.2) using Hisat2 [58] (parameters: --rna-strandness RF --minintronlen 20 -max-intronlen 10000). Mapped read count of each gene was calculated using HTSeq [71] with options (-s no, -a 10, -m union) and differential expression analyses were performed using EdgeR v3.2.4 [72]. A transcript was identified as differentially expressed in a pairwise comparison if the following criteria were met: false discovery rate (FDR) $\leq 0.001$ and fold change 2 2.0. FPKM values were calculated using Cufflinks packages v2.2.1 [73] and used to generate for multidimensional scaling (MDS) plots and gene expression heatmaps.

\section{References}

500

1. Ijima I: On a New Cestode Larva Parasitic in Man (Plerocercoides Prolifer). 1905.

501

2. Stiles $\mathrm{CW}$ : The occurrence of a proliferating cestode larva (Sparganum proliferum) in man in Florida. Hyg Lab Bull 1908, 40:7-18.

503

3. Meric R, Ilie MI, Hofman V, Rioux-Leclercq N, Michot L, Haffaf $Y$, Nelson AM, Neafie

504

505

506

507

508

509

510 RC, Hofman P: Disseminated infection caused by Sparganum proliferum in an AIDS patient. Histopathology 2010, 56:824-828.

4. Nakamura T, Hara M, Matsuoka M, Kawabata M, Tsuji M: Human proliferative sparganosis: a new Japanese case. American journal of clinical pathology 1990, 94:224-228.

5. Kikuchi T, Maruyama H: Human proliferative sparganosis update. Parasitology International 2019:102036.

6. Iwata S: On the branched plerocercoid (Sparganum proliferum) from Japanese snake. Prog Med Parasitol Jpn 1972, 4:587-590.

7. Mueller JF, Strano AJ: Sparganum proliferum, a sparganum infected with a virus? The Journal of parasitology 1974:15-19.

8. Kokaze A, Miyadera H, Kita K, Machinami R, Noya O, de Noya BA, Okamoto M, Horii T, Kojima S: Phylogenetic identification of Sparganum proliferum as a pseudophyllidean cestode. Parasitology International 1997, 46:271-279.

9. Miyadera H, Kokaze A, Kuramochi T, Kita K, Machinami R, Noya O, de Noya BA, Okamoto M, Kojima S: Phylogenetic identification of Sparganum proliferum as a 
pseudophyllidean cestode by the sequence analyses on mitochondrial $\mathrm{COI}$ and nuclear sdhB genes. Parasitology international 2001, 50:93-104.

10. Reuter $\mathrm{M}$, Kreshchenko $\mathrm{N}$ : Flatworm asexual multiplication implicates stem cells and regeneration. Canadian Journal of Zoology 2004, 82:334-356.

11. Specht D, Voge M: ASEXUAL MULTIPLICATION OF MESOCESTOIDES TETRATHYRIDIA IN LABORATORY ANIMALS. J Parasitol 1965, 51:268-272.

12. Bennett HM, Mok HP, Gkrania-Klotsas E, Tsai IJ, Stanley EJ, Antoun NM, Coghlan A, Harsha B, Traini A, Ribeiro DM: The genome of the sparganosis tapeworm Spirometra erinaceieuropaei isolated from the biopsy of a migrating brain lesion. Genome biology 2014, 15:510.

13. Almeida GG, Coscarelli D, Melo MN, Melo AL, Pinto HA: Molecular identification of Spirometra spp. (Cestoda: Diphyllobothriidae) in some wild animals from Brazil. Parasitol Int 2016, 65:428-431.

14. Jeon HK, Park H, Lee D, Choe S, Kim KH, Sohn WM, Eom KS: Genetic Identification of Spirometra decipiens Plerocercoids in Terrestrial Snakes from Korea and China. Korean J Parasitol 2016, 54:181-185.

15. Bernt M, Bleidorn C, Braband A, Dambach J, Donath A, Fritzsch G, Golombek A, Hadrys $H$, Juhling $F$, Meusemann $K$, et al: A comprehensive analysis of bilaterian mitochondrial genomes and phylogeny. Mol Phylogenet Evol 2013, 69:352-364.

16. Tsai IJ, Zarowiecki M, Holroyd N, Garciarrubio A, Sanchez-Flores A, Brooks KL, Tracey $A$, Bobes RJ, Fragoso $G$, Sciutto $E$, et al: The genomes of four tapeworm species reveal adaptations to parasitism. Nature 2013, 496:57-63.

17. Lahti DC, Johnson NA, Ajie BC, Otto SP, Hendry AP, Blumstein DT, Coss RG, Donohue K, Foster SA: Relaxed selection in the wild. Trends Ecol Evol 2009, 24:487-496.

18. Cebria F, Newmark PA: Morphogenesis defects are associated with abnormal nervous system regeneration following roboA RNAi in planarians. Development 2007, 134:833-837.

19. Basseres DS, Tizzei EV, Duarte AA, Costa FF, Saad ST: ARHGAP10, a novel human gene coding for a potentially cytoskeletal Rho-GTPase activating protein. Biochem Biophys Res Commun 2002, 294:579-585.

20. Wenemoser D, Lapan SW, Wilkinson AW, Bell GW, Reddien PW: A molecular wound response program associated with regeneration initiation in planarians. Genes Dev 2012, 26:988-1002.

21. Lei K, Thi-Kim Vu H, Mohan RD, McKinney SA, Seidel CW, Alexander R, Gotting K, Workman JL, Sanchez Alvarado A: Egf Signaling Directs Neoblast Repopulation by Regulating Asymmetric Cell Division in Planarians. Dev Cell 2016, 38:413-429.

22. Klemba M, Goldberg DE: Biological roles of proteases in parasitic protozoa. Annu Rev Biochem 2002, 71:275-305.

23. Frees D, Brondsted L, Ingmer H: Bacterial proteases and virulence. Subcell Biochem 2013, 66:161-192.

24. Yike I: Fungal proteases and their pathophysiological effects. Mycopathologia 2011, 171:299-323.

25. Coleman JJ, Mylonakis E: Efflux in Fungi: La Pièce de Résistance. PLOS Pathogens 2009, 5:e1000486.

26. Florin L, Lang T: Tetraspanin Assemblies in Virus Infection. Front Immunol 2018, 9:1140. 
27. Hunt VL, Hino A, Yoshida A, Kikuchi T: Comparative transcriptomics gives insights into the evolution of parasitism in Strongyloides nematodes at the genus, subclade and species level. Sci Rep 2018, 8:5192.

28. Hunt VL, Tsai IJ, Coghlan A, Reid AJ, Holroyd N, Foth BJ, Tracey A, Cotton JA, Stanley $E J$, Beasley $H$, et al: The genomic basis of parasitism in the Strongyloides clade of nematodes. Nat Genet 2016, 48:299-307.

29. Zheng H, Zhang W, Zhang L, Zhang Z, Li J, Lu G, Zhu Y, Wang Y, Huang Y, Liu J, et al: The genome of the hydatid tapeworm Echinococcus granulosus. Nat Genet 2013, 45:1168-1175.

30. Li W, Liu B, Yang Y, Ren Y, Wang S, Liu C, Zhang N, Qu Z, Yang W, Zhang Y, et al: The genome of tapeworm Taenia multiceps sheds light on understanding parasitic mechanism and control of coenurosis disease. DNA Res 2018, 25:499-510.

31. International Helminth Genomes C: Comparative genomics of the major parasitic worms. Nature genetics 2019, 51:163-174.

32. Kondo N, Nikoh N, ljichi N, Shimada M, Fukatsu T: Genome fragment of Wolbachia endosymbiont transferred to $\mathrm{X}$ chromosome of host insect. Proc Natl Acad Sci U S A 2002, 99:14280-14285.

33. Aikawa T, Anbutsu H, Nikoh N, Kikuchi T, Shibata F, Fukatsu T: Longicorn beetle that vectors pinewood nematode carries many Wolbachia genes on an autosome. Proc Biol Sci 2009, 276:3791-3798.

34. Walsh AM, Kortschak RD, Gardner MG, Bertozzi T, Adelson DL: Widespread horizontal transfer of retrotransposons. Proc Natl Acad Sci U S A 2013, 110:1012-1016.

35. Ivancevic AM, Kortschak RD, Bertozzi T, Adelson DL: Horizontal transfer of BovB and L1 retrotransposons in eukaryotes. Genome Biol 2018, 19:85.

36. Carroll SB: Homeotic genes and the evolution of arthropods and chordates. Nature 1995, 376:479-485.

37. Moens CB, Selleri L: Hox cofactors in vertebrate development. Dev Biol 2006, 291:193-206.

38. Olson PD, Zarowiecki M, James K, Baillie A, Bartl G, Burchell P, Chellappoo A, Jarero F, Tan LY, Holroyd N, Berriman M: Genome-wide transcriptome profiling and spatial expression analyses identify signals and switches of development in tapeworms. Evodevo 2018, 9:21.

39. Gold DA, Gates RD, Jacobs DK: The early expansion and evolutionary dynamics of POU class genes. Mol Biol Evol 2014, 31:3136-3147.

40. Dirgahayu P, Fukumoto S, Tademoto S, Kina Y, Hirai K: Excretory/secretory products from plerocercoids of Spirometra erinaceieuropaei suppress interleukin-1beta gene expression in murine macrophages. Int J Parasitol 2004, 34:577-584.

41. Kina Y, Fukumoto S, Miura K, Tademoto S, Nunomura K, Dirgahayu P, Hirai K: A glycoprotein from Spirometra erinaceieuropaei plerocercoids suppresses osteoclastogenesis and proinflammatory cytokine gene expression. Int J Parasitol 2005, 35:1399-1406.

42. Sakai T, Larsen M, Yamada KM: Fibronectin requirement in branching morphogenesis. Nature 2003, 423:876-881.

43. Wang S, Sekiguchi R, Daley WP, Yamada KM: Patterned cell and matrix dynamics in branching morphogenesis. The Journal of Cell Biology 2017, 216:559-570. 
44. Moulinier R, Martinez E, Torres J, Noya O, de Noya BA, Reyes O: Human proliferative sparganosis in Venezuela: report of a case. The American journal of tropical medicine and hygiene 1982, 31:358-363.

45. Alarcon de Noya B, Torres JR, Noya O: Maintenance of Sparganum proliferum in vitro and in experimental animals. Int J Parasitol 1992, 22:835-838.

46. Kokot M, Dlugosz M, Deorowicz S: KMC 3: counting and manipulating k-mer statistics. Bioinformatics 2017, 33:2759-2761.

47. Vurture GW, Sedlazeck FJ, Nattestad M, Underwood CJ, Fang H, Gurtowski J, Schatz MC: GenomeScope: fast reference-free genome profiling from short reads. Bioinformatics 2017, 33:2202-2204.

48. Jaron KS, Bast J, Ranallo-Benavidez TR, Robinson-Rechavi M, Schwander T: Genomic features of asexual animals. BioRxiv 2018:497495.

49. Kajitani R, Toshimoto K, Noguchi H, Toyoda A, Ogura Y, Okuno M, Yabana M, Harada $M$, Nagayasu $E$, Maruyama $H$ : Efficient de novo assembly of highly heterozygous genomes from whole-genome shotgun short reads. Genome research 2014, 24:13841395.

50. Huang $S$, Kang $M, X u A$ : HaploMerger2: rebuilding both haploid sub-assemblies from high-heterozygosity diploid genome assembly. Bioinformatics 2017, 33:2577-2579.

51. Boetzer M, Henkel CV, Jansen HJ, Butler D, Pirovano W: Scaffolding pre-assembled contigs using SSPACE. Bioinformatics 2010, 27:578-579.

52. Parra G, Bradnam K, Korf I: CEGMA: a pipeline to accurately annotate core genes in eukaryotic genomes. Bioinformatics 2007, 23:1061-1067.

53. Simão FA, Waterhouse RM, loannidis $P$, Kriventseva EV, Zdobnov EM: BUSCO: assessing genome assembly and annotation completeness with single-copy orthologs. Bioinformatics 2015, 31:3210-3212.

54. Hahn C, Bachmann L, Chevreux B: Reconstructing mitochondrial genomes directly from genomic next-generation sequencing reads--a baiting and iterative mapping approach. Nucleic Acids Res 2013, 41:e129.

55. Bernt M, Donath A, Juhling F, Externbrink F, Florentz C, Fritzsch G, Putz J, Middendorf $M$, Stadler PF: MITOS: improved de novo metazoan mitochondrial genome annotation. Mol Phylogenet Evol 2013, 69:313-319.

56. Carver T, Harris SR, Berriman M, Parkhill J, McQuillan JA: Artemis: an integrated platform for visualization and analysis of high-throughput sequence-based experimental data. Bioinformatics 2012, 28:464-469.

57. Stanke M, Waack S: Gene prediction with a hidden Markov model and a new intron submodel. Bioinformatics 2003, 19:ii215-ii225.

58. Kim D, Langmead B, Salzberg SL: HISAT: a fast spliced aligner with low memory requirements. Nat Methods 2015, 12:357-360.

59. El-Gebali S, Mistry J, Bateman A, Eddy SR, Luciani A, Potter SC, Qureshi M, Richardson $\amalg$, Salazar GA, Smart A: The Pfam protein families database in 2019. Nucleic acids research 2018, 47:D427-D432.

60. Gotz S, Garcia-Gomez JM, Terol J, Williams TD, Nagaraj SH, Nueda MJ, Robles M, Talon $\mathrm{M}$, Dopazo J, Conesa A: High-throughput functional annotation and data mining with the Blast2GO suite. Nucleic Acids Res 2008, 36:3420-3435.

61. Katoh $K$, Standley DM: MAFFT multiple sequence alignment software version 7: improvements in performance and usability. Molecular biology and evolution 2013, 30:772-780. 
62. Castresana J: Selection of conserved blocks from multiple alignments for their use in phylogenetic analysis. Mol Biol Evol 2000, 17:540-552.

63. Stamatakis A: RAxML version 8: a tool for phylogenetic analysis and post-analysis of large phylogenies. Bioinformatics 2014, 30:1312-1313.

64. Emms DM, Kelly S: OrthoFinder: solving fundamental biases in whole genome comparisons dramatically improves orthogroup inference accuracy. Genome biology 2015, 16:157.

65. De Bie T, Cristianini N, Demuth JP, Hahn MW: CAFE: a computational tool for the study of gene family evolution. Bioinformatics 2006, 22:1269-1271.

66. Podell S, Gaasterland T: DarkHorse: a method for genome-wide prediction of horizontal gene transfer. Genome Biol 2007, 8:R16.

67. Huerta-Cepas J, Serra F, Bork P: ETE 3: reconstruction, analysis, and visualization of phylogenomic data. Molecular biology and evolution 2016, 33:1635-1638.

68. Yang Z: PAML: a program package for phylogenetic analysis by maximum likelihood. Bioinformatics 1997, 13:555-556.

69. Suyama $M$, Torrents $D$, Bork P: PAL2NAL: robust conversion of protein sequence alignments into the corresponding codon alignments. Nucleic acids research 2006, 34:W609-W612.

70. Wertheim JO, Murrell B, Smith MD, Kosakovsky Pond SL, Scheffler K: RELAX: detecting relaxed selection in a phylogenetic framework. Mol Biol Evol 2015, 32:820-832.

71. Anders S, Pyl PT, Huber W: HTSeq-a Python framework to work with highthroughput sequencing data. Bioinformatics 2015, 31:166-169.

72. Robinson MD, McCarthy DJ, Smyth GK: edgeR: a Bioconductor package for differential expression analysis of digital gene expression data. Bioinformatics 2010, 26:139-140.

73. Trapnell C, Roberts A, Goff L, Pertea G, Kim D, Kelley DR, Pimentel H, Salzberg SL, Rinn $\mathrm{JL}$, Pachter L: Differential gene and transcript expression analysis of RNA-seq experiments with TopHat and Cufflinks. Nature protocols 2012, 7:562.

Declarations

Ethics approval and consent to participate: All animal experiments in this study were performed under the applicable laws and guidelines for the care and use of laboratory animals, as specified in the Fundamental Guidelines for Proper Conduct of Animal Experiment and Related Activities in Academic Research Institutions under the jurisdiction of the Ministry of Education, Culture, Sports, Science and Technology, Japan, 2006.

Consent for publication: Not applicable.

Availability of data and materials: All sequence data from the genome projects have been deposited at DDBJ/ENA/GenBank under BioProject accession PRJEB35374 and PRJEB35375. All relevant data are available from the authors.

Competing interests: The authors declare that they have no competing interests

Funding: This work was supported by Japan Society for the Promotion of Science (JSPS) KAKENHI Grant Numbers 26460510 and 19H03212, AMED 18fk0108009h0003 and JST CREST Grant Number JPMJCR18S7.

Authors' Contributions: T.Ki., T.Ku. and H.M. conceived the study. T.Ki. contributed to study design. V.L.H., H.M. and T.Ki. wrote the manuscript with inputs from others. BAN, ON, SK prepared 
biological samples. Ki and T.Ku. conducted experiments. V.L.H., M.D., Y.M., A.T. and T.Ki. completed genome assembly and analysed genome data.

705 Acknowledgements: Genome data analyses were partly performed using the DDBJ supercomputer system. We thank Ryusei Tanaka, Asuka Kounosu, Akemi Yoshida for assistance and comments.

707 Author Information Correspondence and requests for materials should be addressed to T.K. (taisei kikuchi@med.miyazaki-u.ac.jp).

709

710 
711

Table 1. Statistics of genome assemblies

\begin{tabular}{|c|c|c|c|c|c|c|c|c|}
\hline & $\begin{array}{l}\text { Sparganu } \\
\text { m } \\
\text { proliferum } \\
\text { (v2.2) }\end{array}$ & $\begin{array}{l}\text { Spirometra } \\
\text { erinaceieur } \\
\text { opaei } \\
\text { (v2.0) }\end{array}$ & $\begin{array}{l}\text { Spirometra } \\
\text { erinaceieur } \\
\text { opaei (UK) } \\
\text { (WBPS13) }\end{array}$ & $\begin{array}{l}\text { Diphyllobot } \\
\text { hrium } \\
\text { latum } \\
\text { (WBPS13) }\end{array}$ & $\begin{array}{l}\text { Schistocep } \\
\text { halus } \\
\text { solidus } \\
\text { (WBPS13) }\end{array}$ & $\begin{array}{c}\text { Hymenolep } \\
\text { is } \\
\text { microstom } \\
\text { a } \\
\text { (WBPS13) }\end{array}$ & $\begin{array}{c}\text { Taenia } \\
\text { solium } \\
\text { (WBPS13) }\end{array}$ & $\begin{array}{c}\text { Echinococc } \\
\text { us } \\
\text { multilocular } \\
\text { is } \\
\text { (WBPS13) }\end{array}$ \\
\hline Assembly size (Mb) & 653.4 & 796.0 & 1258.7 & 531.4 & 539.4 & 182.1 & 122.4 & 114.5 \\
\hline Num. scaffolds & 7,388 & 5,723 & 482,608 & 140,336 & 56,778 & 3,643 & 11,237 & 1,288 \\
\hline Average (kb) & 88.4 & 139.0 & 2.6 & 3.8 & 10.0 & 50.0 & 11.2 & 889.3 \\
\hline Largest scaff (kb) & 8,099 & 5,490 & 90 & 80 & 595 & 2,234 & 740 & 15,981 \\
\hline N50 (kb) & 1,242 & 821 & 5 & 7 & 32 & 767 & 68 & 5,229 \\
\hline N90 (kb) & 110 & 167 & 1 & 2 & 5 & 41 & 5 & 213 \\
\hline Gaps (kb) & 51,020 & 77,788 & 128,163 & 38,407 & 22,091 & 259 & 164 & 336 \\
\hline $\begin{array}{l}\text { CEGMA completeness } \\
\text { complete/partial (\%) }\end{array}$ & $61.7 / 81.5$ & $58.5 / 80.2$ & $29.4 / 45.9$ & $49.6 / 65.3$ & $76.6 / 87.9$ & $91.9 / 92.7$ & $87.1 / 90.7$ & $93.2 / 93.2$ \\
\hline $\begin{array}{l}\text { Average CEG number } \\
\text { complete/partial }\end{array}$ & $1.1 / 1.2$ & $1.1 / 1.3$ & $1.8 / 2.2$ & $1.5 / 1.6$ & $1.2 / 1.3$ & $1.1 / 1.1$ & $1.2 / 1.2$ & $1.1 / 1.1$ \\
\hline $\begin{array}{c}\text { BUSCO completeness } \\
\text { (Metazoa } \\
\text { dataset/Eukaryota dataset) }\end{array}$ & $72.0 / 88.1$ & $71.9 / 88.5$ & $33.6 / 37.3$ & $38.1 / 53.8$ & $70.3 / 86.2$ & $78.6 / 90.4$ & $72.6 / 85.5$ & $72.2 / 88.1$ \\
\hline Num. coding genes & 25,627 & 30,751 & 39,557 & 19,966 & 20,228 & 12,373 & 12,481 & 10,273 \\
\hline $\begin{array}{l}\text { Coding gene size (median; } \\
\text { aa) }\end{array}$ & 665.0 & 627.0 & 200.0 & 216.0 & 455.0 & 709.0 & 609.0 & 596.0 \\
\hline
\end{tabular}

712 
Table 2. Statistics of repeats in genomes

\begin{tabular}{l|rrrr}
\hline & \multicolumn{3}{c}{ Sparganum proliferum (v2.2) } & $\begin{array}{c}\text { Spirometra erinaceieuropaei } \\
\text { (v2) }\end{array}$ \\
\hline & num element & \% in bp & num element & \% in bp \\
\hline SINEs: & 49435 & 1.59 & 45184 & 1.09 \\
LINEs: & & & & \\
LINE/Penelope & 390951 & 26.32 & 519275 & 31.90 \\
LINE/RTE-BovB & 139623 & 7.95 & 214116 & 10.41 \\
LINE/CR1 & 162469 & 10.24 & 188656 & 11.18 \\
& 75037 & 7.59 & 101503 & 9.37 \\
LTR element:1 & & & & \\
LTR/Gypsy & 18276 & 1.79 & 25374 & 1.88 \\
& 16179 & 1.56 & 24544 & 1.81 \\
DNA element: & & & & \\
DNA/CMC-EnSpm & 22386 & 1.38 & 54802 & 2.48 \\
DNA/TcMar-Tc1 & 5795 & 0.35 & 16161 & 0.69 \\
& 8162 & 0.59 & 7416 & 0.53 \\
Small RNA: & & & & \\
Satellites: & 2906 & 0.15 & 2955 & 0.08 \\
Simple repeat: & 10962 & 0.39 & 5823 & 0.16 \\
Low complexity: & 79986 & 1.21 & 68909 & 0.76 \\
Unclassified: & 3799 & 0.04 & 5690 & 0.06 \\
\hline TOTAL & 378820 & 20.68 & 425608 & 15.92 \\
\hline & 1004498 & & 1185136 & \\
& $(55.01 \%)$ & & $(55.14 \%)$ & \\
& & & &
\end{tabular}


719 Table 3. Homeobox complement in S. proliferum and S. erinaceieuropaei compared with other tapeworms and bilaterians

\begin{tabular}{lrrrrr}
\hline homeobox class & $\begin{array}{c}\text { Sparganum } \\
\text { proliferum } \\
\text { (v2.2) }\end{array}$ & $\begin{array}{c}\text { Spirometra } \\
\text { erinaceieuropaei } \\
\text { (v2.0) }\end{array}$ & $\begin{array}{c}\text { Taenia solium } \\
\text { (WBPS13) }\end{array}$ & $\begin{array}{c}\text { Echinococcus } \\
\text { multilocularis } \\
\text { (WBPS13) }\end{array}$ & $\begin{array}{c}\text { Branchiostoma } \\
\text { floridae }\end{array}$ \\
\hline ANTP & 25 & 30 & 36 & 25 & 58 \\
PRD & 10 & 8 & 11 & $15(18)$ & 21 \\
CUT & 3 & 4 & 3 & 3 & 4 \\
SINE & 3 & 4 & 2 & 3 & 3 \\
TALE & 8 & 10 & 11 & 12 & 10 \\
CERS & 2 & 1 & 2 & 2 & 1 \\
POU & 3 & 4 & 4 & 5 & 6 \\
LIM & 6 & 6 & 7 & 7 & 8 \\
ZF & 4 & 4 & $3(2)$ & $3(2)$ & 4 \\
\hline Total & 64 & 71 & 79 & 75 & 115
\end{tabular}




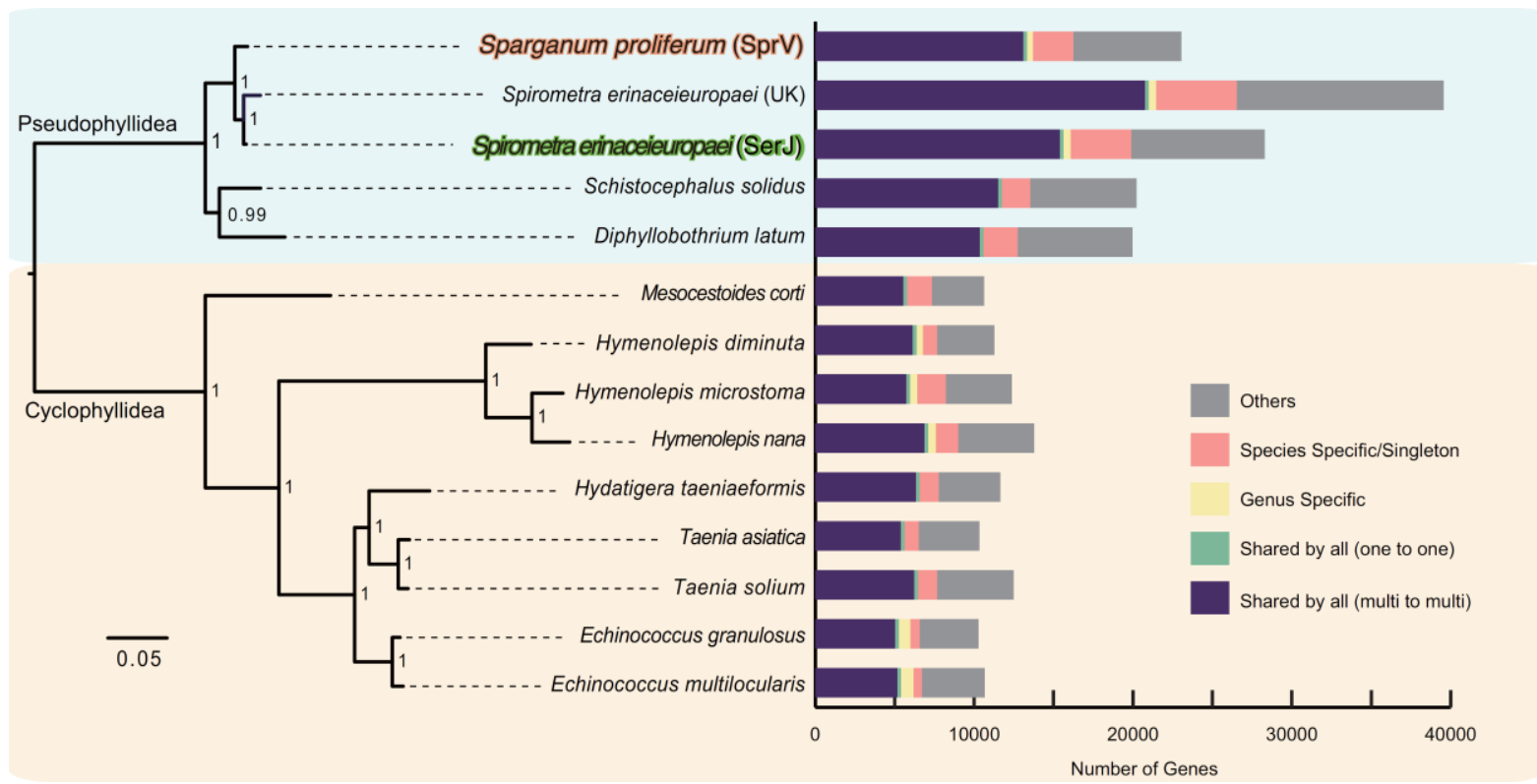

724 Figure 1. Phylogeny and gene contents; genes are categorized in a stack bar, and the length of 725 stack bar is proportional to number of genes. 


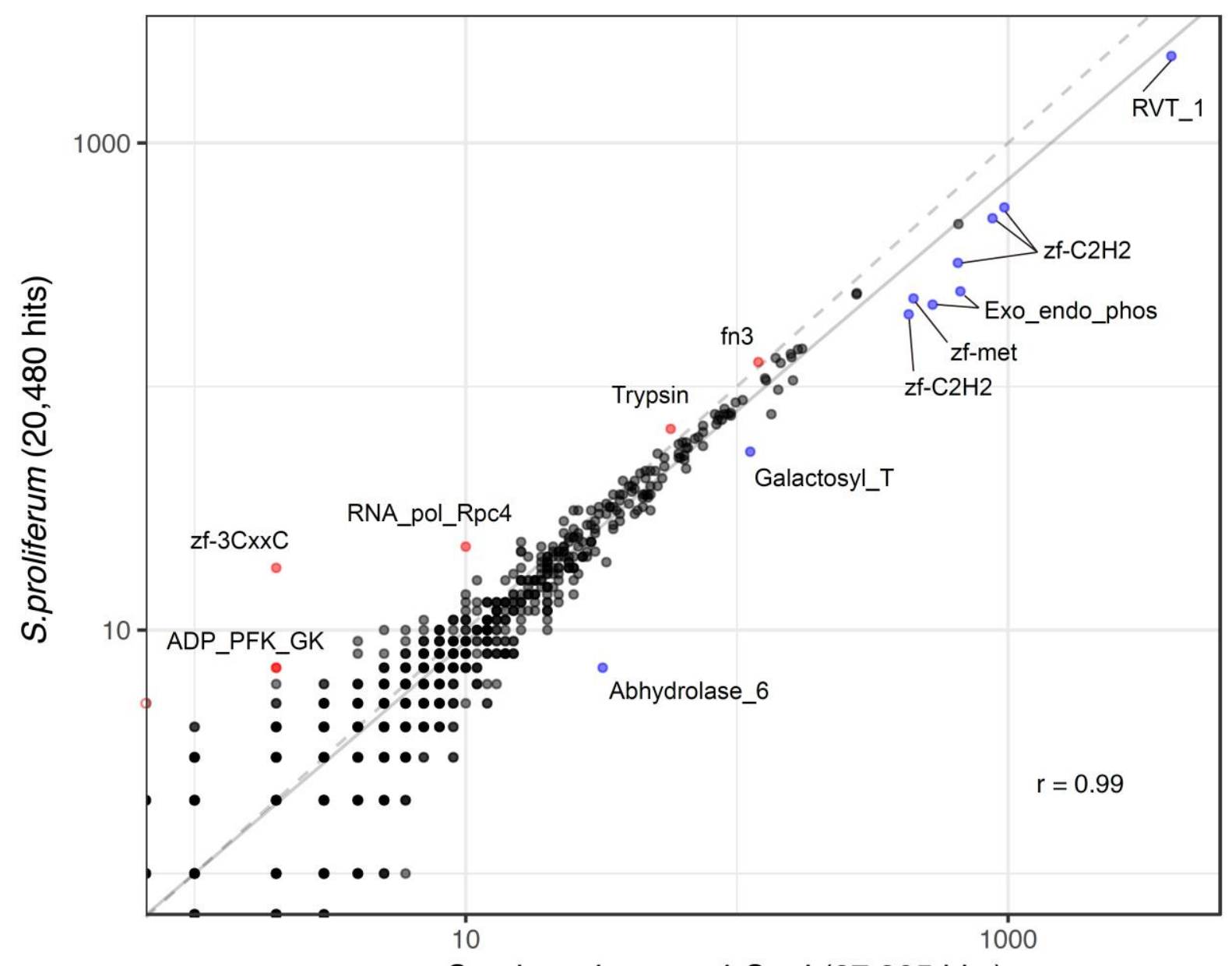

729 Figure 2. A scatterplot showing the abundance of Pfam domains in S. proliferum and $S$. 730 erinaceieuropaei genomes; Pfam domains that are more enriched in $S$. proliferum than in $S$. 731 erinaceieuropaei are highlighted in red. Those enriched in $S$. erinaceieuropaei relative to $S$. 732 proliferum are highlighted in blue. 
Gene gain/loss

Family expansion/contraction $\quad+7298 /-2337$

$+665 /-2098$

Sparganum proliferum (SprV)

$+393 /-2474$

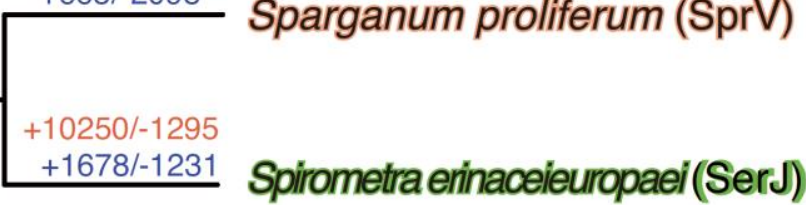

$+1781 /-2903$

$+1678 /-123$

$+652 /-2856$

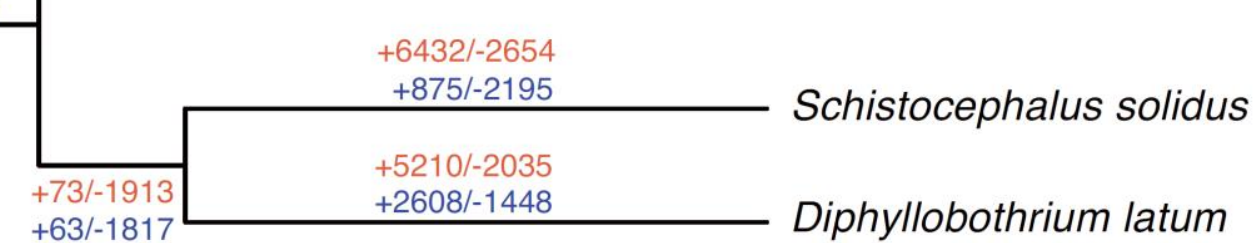

738

739 Figure 3. Gene family evolution of selected cestode species was inferred using computational

740 analysis of gene family evolution (CAFE). Numbers on each branch (or lineage) indicate specific

741 gains/losses of that branch (or lineage).

742 

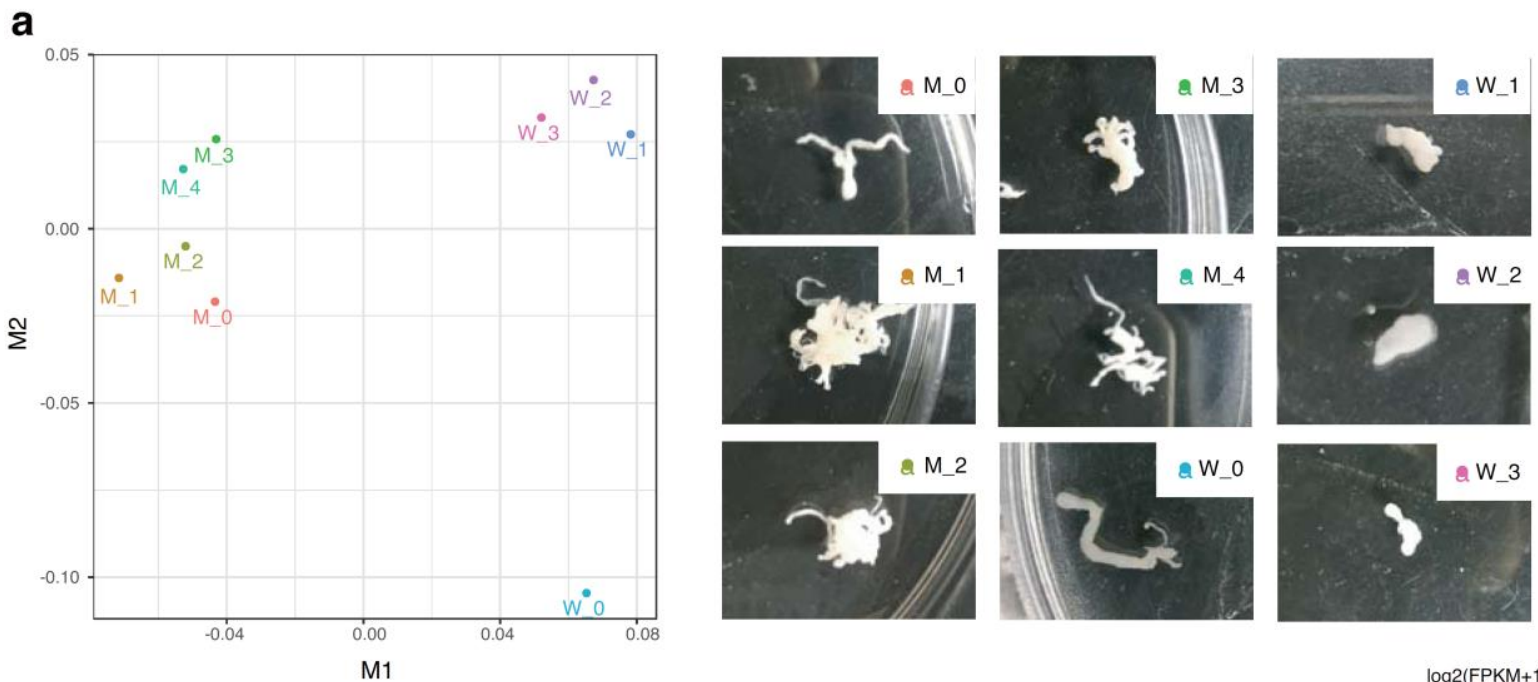

b
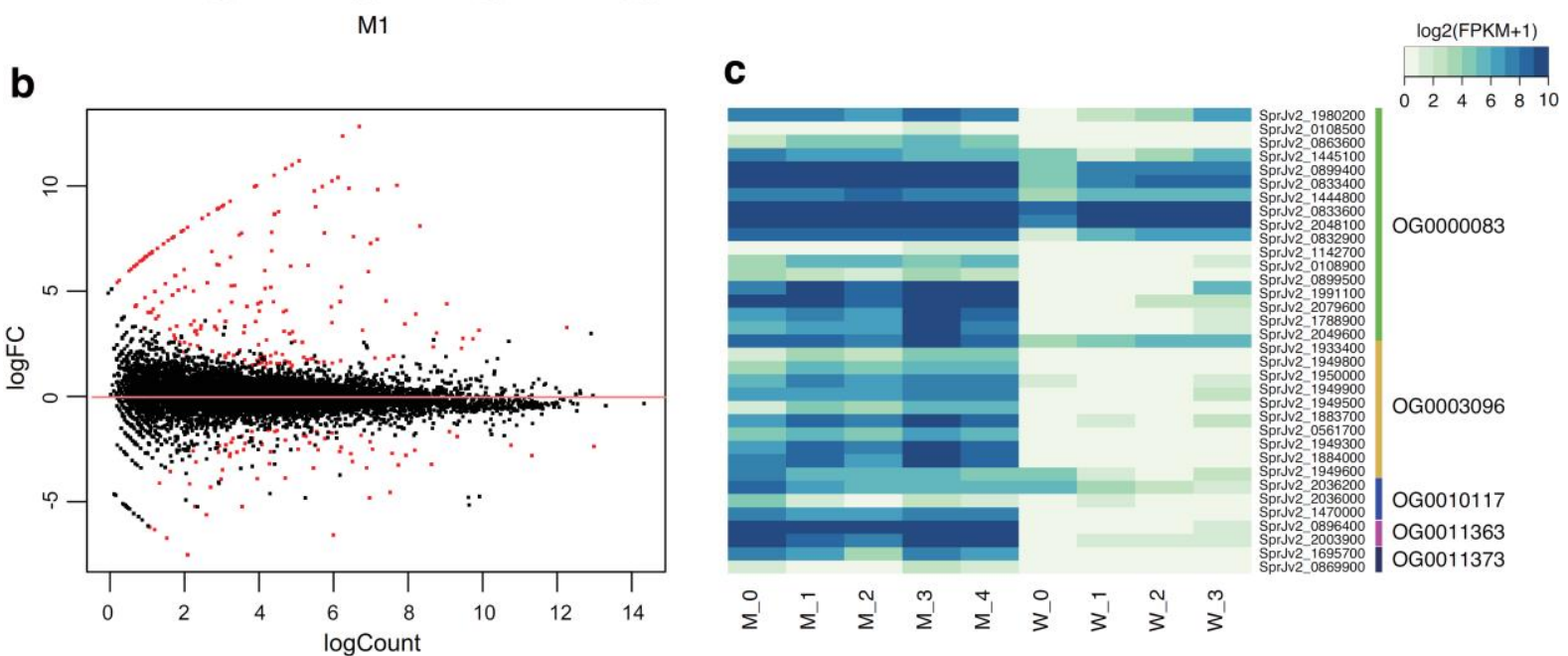

C

745 Figure 4. Comparison of gene expression in highly branching worms (medusa-head form) relative

746 to static worms (wasabi-root form) of S. proliferum: A) multidimensional scaling (MDS) analyses of

747 RNA-seq samples clearly separate the two forms by dimension 1. Pictures of used samples are

748 shown on the right. B) Bland-Altman (MA) plot of the two-form comparison; dots represent

749 transcripts and log2 fold changes (medusa-head/wasabi-root) plotted against average abundance

750 in counts per million. Red dots indicate differentially expressed transcripts with false discovery

751 rates (FDR) of $<0.05$ and fold changes of $>2$. C) Heatmap of gene families encoding novel 752 secreted proteins; the heat map shows log2 fragments per kilobase per million reads mapped

753 (FPKM) values for 5 gene families. 
Figures

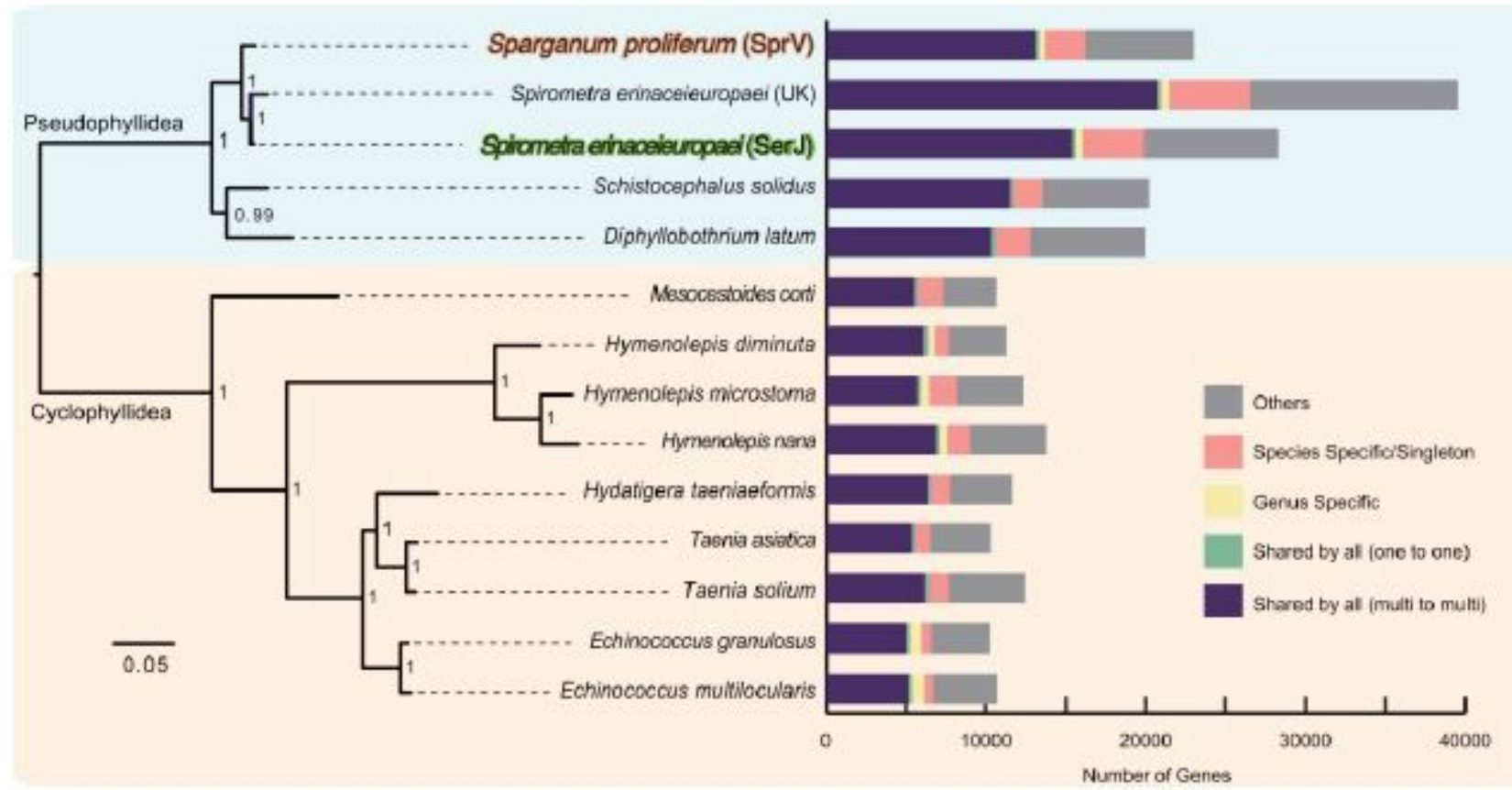

Figure 1

Phylogeny and gene contents; genes are categorized in a stack bar, and the length of stack bar is proportional to number of genes. 


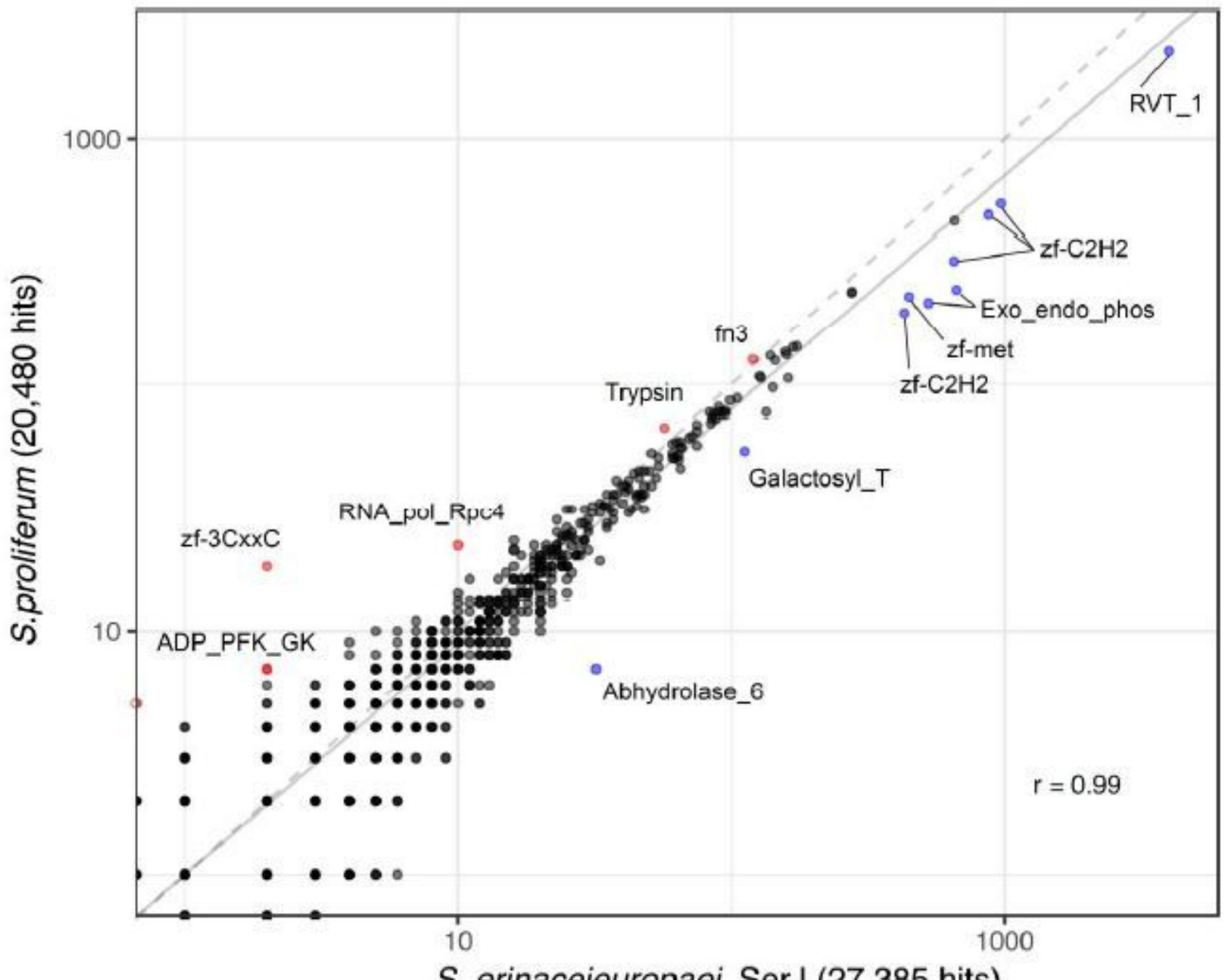

S. erinaceieuropaei SerJ (27,385 hits)

Figure 2

A scatterplot showing the abundance of Pfam domains in S. proliferum and S. erinaceieuropaei genomes; Pfam domains that are more enriched in S. proliferum than in S. erinaceieuropaei are highlighted in red. Those enriched in S. erinaceieuropaei relative to S. proliferum are highlighted in blue. 


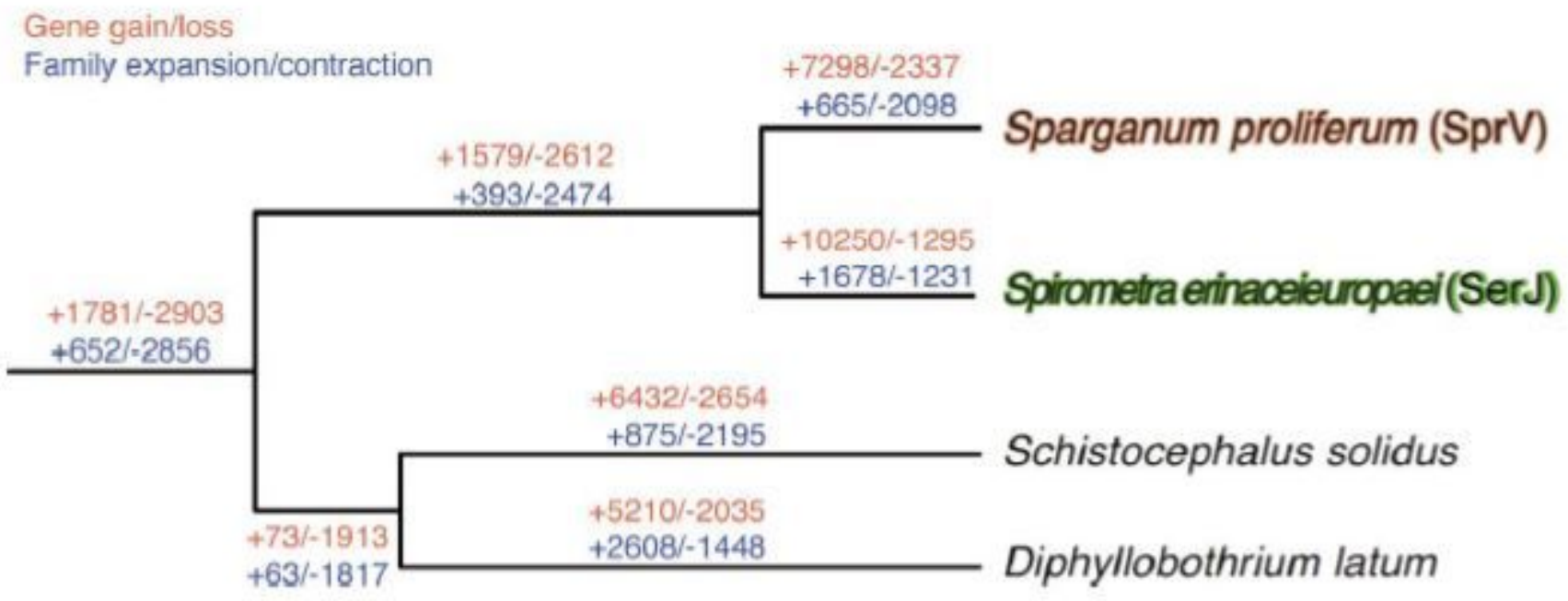

\section{Figure 3}

Gene family evolution of selected cestode species was inferred using computational analysis of gene family evolution (CAFE). Numbers on each branch (or lineage) indicate specific gains/losses of that branch (or lineage). 

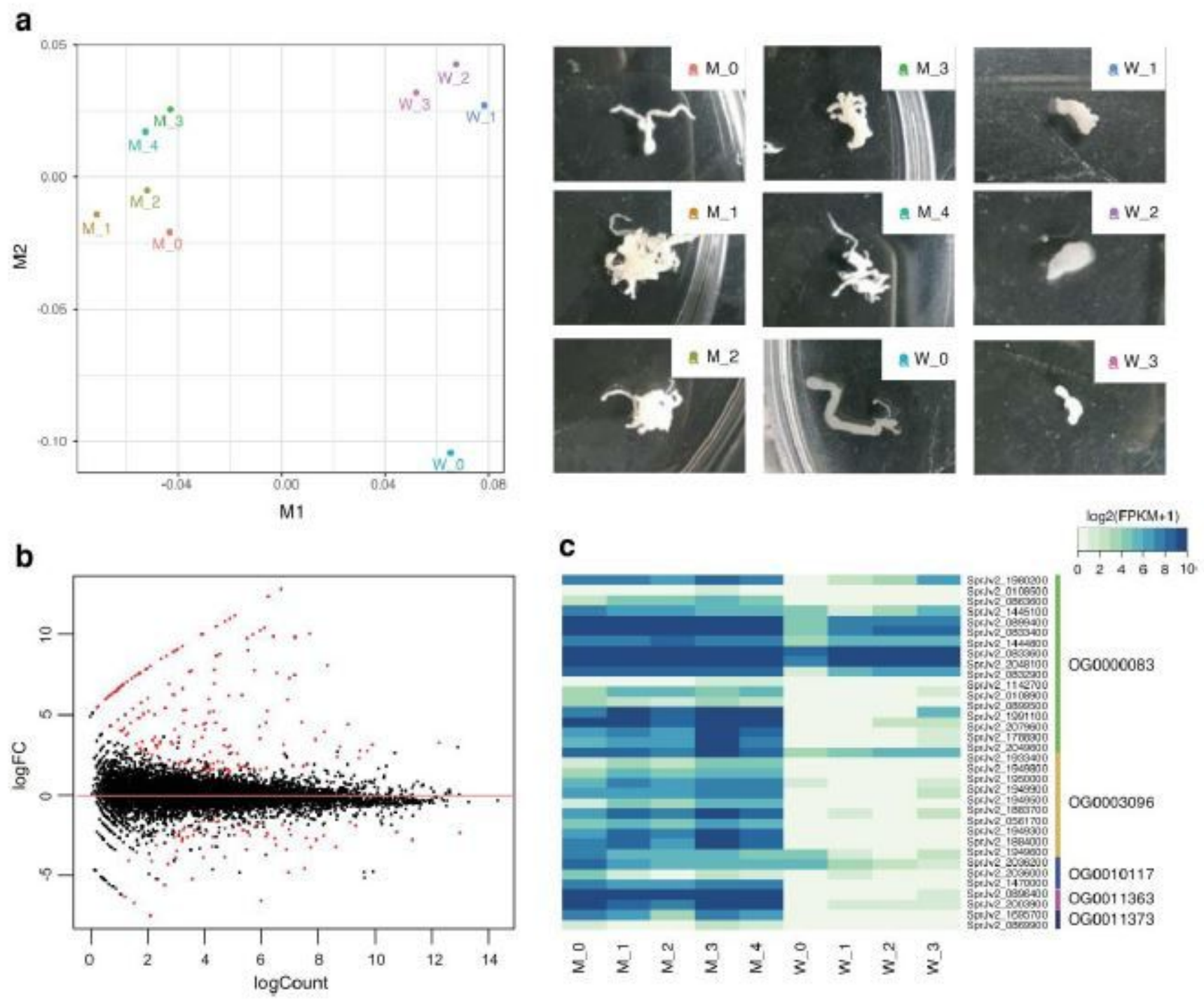

\section{Figure 4}

Comparison of gene expression in highly branching worms (medusa-head form) relative to static worms (wasabi-root form) of S. proliferum: A) multidimensional scaling (MDS) analyses of RNA-seq samples clearly separate the two forms by dimension 1. Pictures of used samples are shown on the right. B) Bland-Altman (MA) plot of the two-form comparison; dots represent transcripts and log2 fold changes (medusa-head/wasabi-root) plotted against average abundance in counts per million. Red dots indicate differentially expressed transcripts with false discovery rates (FDR) of $<0.05$ and fold changes of $>2$. C) Heatmap of gene families encoding novel secreted proteins; the heat map shows log2 fragments per kilobase per million reads mapped (FPKM) values for 5 gene families.

\section{Supplementary Files}

This is a list of supplementary files associated with this preprint. Click to download.

- supplement1.pdf 
- supplement2.xlsx 